\title{
Dispersão de sementes de Melocactus glaucescens e M. paucispinus (Cactaceae), no Município de Morro do Chapéu, Chapada Diamantina - BA
}

\author{
Rosineide Braz Santos Fonseca ${ }^{1,4}$, Ligia Silveira Funch ${ }^{2}$ e Eduardo Leite Borba ${ }^{3}$
}

Recebido em 17/04/2011. Aceito em 13/04/2012

\section{RESUMO}

(Dispersão de sementes de Melocactus glaucescens e M. paucispinus (Cactaceae), no município de Morro do Chapéu, Chapada Diamantina - BA). Objetivou-se identificar os dispersores de duas espécies de Melocactus, verificar o padrão de liberação dos frutos, correlacionando-o com a remoção por frugívoros e variação térmica do cefálio, bem como caracterizar a distribuição espacial. Para tanto, foram realizadas observações focais, testes de germinação, registros da emergência, extrusão e remoção de frutos, da temperatura do cefálio, de interações formigas-diásporos e distribuição espacial. Lagartos ( 2 espécies) e formigas ( 3 espécies) foram os dispersores das espécies estudadas. A dormência das sementes não foi quebrada pela passagem pelo trato digestivo dos lagartos. As maiores taxas de remoção dos frutos pelos lagartos ocorreram nas horas centrais do dia, coincidindo ou sendo posterior aos picos de extrusão. As taxas de emergência e extrusão foram mais intensas pela manhã, o que aumentou as chances de remoção dos frutos no dia da liberação, evitando dissecação e predação. Não houve correlação entre a liberação dos frutos e a variação térmica do cefálio. O desenvolvimento do fruto gera tensão nas fibras do cefálio que promove sua emergência ou expulsão. A expulsão de frutos emergidos pode ser auxiliada pela dilatação das fibras em resposta ao aquecimento, tensão da união das fibras na base e saída de outros frutos. A distribuição espacial dos indivíduos parece ser influenciada pelo comportamento dos dispersores.

Palavras-chave: dispersão, distribuição espacial, germinação, temperatura do cefálio

\begin{abstract}
(Dispersion of Melocactus glaucescens and M. paucispinus (Cactaceae) in the municipality of Morro do Chapéu, Chapada Diamantina - BA). Objectives: identify the dispersers of two species of Melocactus; verify their patterns of fruit offering and correlate this with removal by frugivores and with temperature variations of the cephalium; and analyze the relationship between the spatial distribution of the Melocactus species and the behavior of their dispersers. Focal observations were made of fruit emergence, extrusion and removal, cephalium temperature, ant-diaspore interactions, and plant spatial distribution. Germination tests were also made. Two lizard and three ant species were dispersers of the two species of Melocactus. Seed dormancy was not broken in the digestive tracts of the lizards. The greatest rates of fruit removal by the lizards occurred during the mid hours of the day, coinciding with or following the extrusion peak. Fruit emergence and extrusion rates were highest in the morning, increasing their chances of being taken on the same day, thus avoiding desiccation and predation. No correlation was observed between fruit liberation and thermal variations of the cephalium. Fruit development generates tension within the fiber mass of the cephalium that provokes fruit emergence/expulsion. The expulsion of the fruits may also be aided by the dilation of the cephalium fibers as they warm, tension generated at the cephalium base where the fibers unite, and by the expulsion of other fruits. The spatial distribution of these cacti is influenced by disperser behavior.
\end{abstract}

Key words: Dispersion, germination, spatial distribution, cephalium temperature

1 Instituto Federal Baiano, Campus Santa Inês, Santa Inês, BA, Brasil

2 Universidade Estadual de Feira de Santana, Departamento de Ciências Biológicas, Feira de Santana, BA, Brasil

3 Universidade Federal do ABC, Centro de Ciências Naturais e Humanas, Santo André, SP, Brasil

4 Autor para correspondência: rosineide_braz@hotmail.com 


\section{Introdução}

O processo de dispersão das angiospermas corresponde ao deslocamento dos diásporos (fruto ou semente) para locais propícios à germinação, cujo transporte pode ser mediado pela ação de agentes abióticos, como água e vento, ou por vetores bióticos, representados geralmente por aves, mamíferos, répteis, peixes e formigas (van der Pijl 1982). O estudo de dispersão, no entanto, não se restringe apenas a observância do processo de dispersão e identificação dos agentes envolvidos. Analisa as características físicas, comportamentais, demográficas e/ou ecológicas das plantas e de seus agentes dispersores relacionadas à dispersão, por exemplo, forma, tamanho e peso dos diásporos, fenologia da frutificação, distribuição espacial das plantas e animais, competição entre plantas e entre frugívoros e a predação de sementes pré- e pós-dispersão (Augspurger 1986, Dirzo \& Dominguez 1986, Jordano 1987, Chapman 1989, Howe 1989, Oliveira \& Moreira 1992, Schupp 1993, Pizo 1997, Pereira \& Mantovani 2001). O estudo da dispersão contribui para entender as estratégias reprodutivas das plantas em diferentes condições ambientais e as inter-relações bióticas envolvidas, constituindo-se em um importante ramo do conhecimento para caracterização e compreensão da dinâmica das populações.

A família Cactaceae, com mais de 1300 espécies (Taylor 2000), possui diásporos predominantemente carnosos, dispersos provavelmente por mamíferos, morcegos, lagartos, formigas e especialmente aves (Ridley 1930, van der Pijl 1982, Bregman 1988, Taylor 2000). O gênero Melocactus possui frutos suculentos, de coloração rosa a vermelho, que se desenvolvem completamente dentro do cefálio, uma estrutura florífera típica, originada da diferenciação do eixo caulinar. Quando maduros os frutos são expostos à superfície do cefálio, tornando-se acessíveis a agentes dispersores, tais como aves, formigas e lagartos (Rizzini 1987, Taylor 1991, Figueira et al. 1994, Taylor 2000, Romão et al. 2007).

Dentre os estudos que caracterizaram a dispersão nesse gênero se destaca o de Figueira et al. (1994), que evidenciou uma série de características de $M$. violaceus Pfeiffer (tamanho pequeno, coloração rósea e padrão de liberação dos frutos), que facilitam a detecção, acesso e remoção dos frutos por lagartos. Mais tarde, em um estudo experimental, foi demonstrado que Tropidurus torquatus utiliza características do fruto e da planta para localização e reconhecimento do fruto de M. violaceus (Vasconcellos-Neto et al. 2000).

O padrão diário de liberação dos frutos, verificado por Figueira et al. (1994), parece ajustado ao padrão de atividade do dispersor, Tropidurus torquatus, e regulado pela temperatura. Esses autores observaram um aumento na taxa de extrusão (expulsão do fruto) com a temperatura, quando os lagartos são mais ativos e inferiram que a temperatura parece governar a emergência (surgimento do fruto na superfície do cefálio) e a extrusão dos frutos, embora não tenha realizado nenhuma aferição desta variável ambiental. Já I. G. Varassin (dados não public.) não observou nenhum padrão após correlacionar à temperatura do cefálio e a extrusão de frutos de $M$. violaceus; a extrusão se iniciou com temperaturas menores, continuando ao longo do dia.

O presente estudo teve como objetivo identificar os dispersores diurnos de Melocactus glaucescens Buining \& Brederoo e $M$ paucispinus G. Heimen \& R. Paul e seus horários e comportamento de forrageio no município de Morro do Chapéu - BA, assim como, verificar o padrão diário de liberação dos frutos e sua correlação com a remoção por frugívoros e a variação térmica do cefálio. Em adição, as populações de $M$. glaucescens e M. paucispinus estudadas foram caracterizadas em relação à sua distribuição espacial.

\section{Materiais e métodos}

\section{Espécies e populações estudadas}

Melocactus glaucescens e $M$. paucispinus são endêmicas da Bahia e possuem status de ameaçadas de extinção na Lista Vermelha da IUCN (M. glaucescens) e no Apêndice I da CITES (M. glaucescens e M. paucispinus) (Taylor 2000, Taylor \& Zappi 2004). Na Lista Oficial do MMA M. glaucescens figura como ameaçada de extinção e M. paucispinus com insuficiência de dados para definir seu status. $M$. glaucescens tem cefálio branco, cladódio glauco quando jovem, flores róseas e frutos vermelho intenso (Taylor 1991, 2000). M. paucispinus caracteriza-se principalmente pelo caule discóide, fortemente depresso, o que é interpretado como uma possível adaptação para minimizar os prejuízos causados por fogo que periodicamente ocorre em áreas de cerrado e campo rupestre (Taylor 1991, 2000), habitats típicos desta espécie.

A população estudada de $M$. glaucescens se encontra a

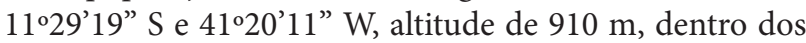
limites do Parque Estadual de Morro do Chapéu. Esta área apresenta clima tropical de altitude de verão quente (Cwa), temperatura média anual de $22^{\circ} \mathrm{C}$ e índice pluviométrico de $700 \mathrm{~mm}$. Os indivíduos de M. glaucescens desenvolvem-se sobre solo arenoso em locais abertos da vegetação, caracterizada como área de tensão fitoecológica, onde a vegetação de caatinga entra em contato com a vegetação de cerrado (CPRM 1995).

A população de $M$. paucispinus estudada se localiza a 11'33'52"S e 41¹0'37” W, a 1104 m de altitude, na porção central de Morro do Chapéu. Esta área apresenta clima tropical de altitude de verão brando $(\mathrm{Cwb})$, temperatura média anual de $19,5^{\circ} \mathrm{C}$ e índice pluviométrico de 700-800 mm. Os indivíduos de M. paucispinus desenvolvem-se sobre solo arenoso em espaços abertos da vegetação, caracterizada como área de tensão fitoecológica, onde a vegetação de cerrado entra em contato com a de campo rupestre, formando mosaicos (CPRM 1995). 


\section{Procedimentos}

Para identificar os dispersores diurnos de M. glaucescens e $M$. paucispinus foram realizadas observações focais, das $06 \mathrm{~h} 00$ às $18 \mathrm{~h} 00$, durante três dias para cada espécie de planta, assim como focais de duração variada toda vez que uma ou mais formigas fossem encontradas explorando os frutos, i.e., em contato com a superfície dos diásporos, extraindo líquidos ou porções destes.

Durante as observações focais realizadas ao longo do dia foram registrados o número de frutos consumidos por espécie de frugívoro, horário em que os frutos foram removidos, comportamento de coleta dos frugívoros e o tratamento dado aos diásporos por estes vertebrados, i.e., se os frutos eram apenas mordidos (sem remoção de partes) ou ingeridos parcialmente ou por completo . Durante as observações focais foram registrados a espécie de formiga, número de formigas por diásporo e/ou indivíduo de planta, horário em que se visualizou a interação e se houve remoção de partes de frutos e/ou sementes. Quando as formigas transportavam as sementes, eram acompanhadas até que as abandonassem, quando então a distância da dispersão era medida.

Os testes de germinação foram realizados com 100 sementes coletadas diretamente da planta mãe (controle) e após passagem pelo trato digestivo das duas espécies de lagartos que foram observadas consumindo os frutos. $\mathrm{O}$ delineamento experimental utilizado foi o inteiramente casualizado. As sementes, após terem sido semi-esterilizadas com hipoclorito de sódio a $0,5 \%$, foram colocadas para germinar em placas de Petri com papel germitest umedecido com água destilada, em câmara de germinação sob fotoperíodo de $12 \mathrm{~h}$ e temperatura controlada de $30^{\circ} \mathrm{C}$. A unidade experimental foi de 25 sementes por placas com quatro repetições. Estas condições de germinação foram estabelecidas após realizar alguns ensaios germinativos que avaliaram o tipo de fotoblastismo das sementes de $M$. glaucescens e M. paucispinus e a temperatura mínima para germinação. As sementes germinadas foram contabilizadas e retiradas da placa diariamente durante um período de 25 dias, sendo a protrusão da radícula o critério adotado para considerar a semente como germinada. As taxas de germinação (\%) das sementes provenientes das fezes dos frugívoros e controle foram transformadas em arco-seno $(\% \mathrm{G} / 100)^{1 / 2}$. As médias foram comparadas pelo teste de Tukey, em nível de $5 \%$ de significância.

Experimentos de germinação realizados paralelamente a este estudo revelaram que $M$. glaucescens apresenta uma dormência fisiológica que é quebrada naturalmente com o tempo, enquanto M. paucispinus parece apresentar uma endodormência vinculada ao período de coleta, i.e., apenas em alguns lotes (Fonseca 2004). Sendo assim, na análise da endozoocoria considerou-se o período de coleta e a idade pós-colheita das sementes. Os frutos de M. glaucescens utilizados para alimentar as espécies de lagartos foram coletados em junho de 2003. Quando o experimento de germinação foi montado, tanto as sementes oriundas das fezes como as do controle (sementes coletadas diretamente da planta mãe) apresentavam idade pós-colheita de 44 dias. Os frutos de M. paucispinus utilizados para alimentar uma das espécies de lagarto (T. cocorobensis) foram coletados em fevereiro de 2003, enquanto os frutos utilizados para alimentar a outra espécie (C. ocellifer) foram coletados em junho de 2003. Quando o experimento de germinação foi montado, as sementes oriundas das fezes de T. cocorobensis, assim como as do controle, apresentavam idade pós-colheita de 200 dias; as sementes das fezes de C. ocellifer, assim como as do controle, apresentavam idade pós-colheita de 44 dias.

Para estimar o padrão diário de liberação dos frutos e de remoção por frugívoro foram realizadas inspeções sucessivas, de hora em hora, entre 06h00 e 18h00, em 30 indivíduos de M. glaucescens e de M. paucispinus, durante dois e três dias, respectivamente, no mês de junho de 2003. Durante as inspeções foram registrados o número de frutos não-disponíveis (emergidos, protraídos até $0,3 \mathrm{~cm}$ do cefálio), o número de frutos disponíveis (estrugidos, protraídos mais que $0,3 \mathrm{~cm}$ a partir do cefálio) e o número de frutos removidos (frutos desaparecidos entre os intervalos de tempo, resíduos temporários de frutos no cefálio e no solo). As taxas de emergência e extrusão foram estimadas como o número de frutos novos emergidos e estrugidos em cada horário inspecionado, respectivamente. A taxa de remoção foi obtida dividindo-se o número de frutos removidos pelo número de frutos disponíveis do horário anterior. Essa metodologia foi similar à utilizada por Figueira et al. (1994), que inspecionaram os indivíduos de M. violaceus de duas em duas horas e consideraram como frutos não-disponíveis aqueles com menos de $1 / 5$ protraídos do cefálio e como disponíveis os frutos com mais de $1 / 4$. Com base nestas proporções e no tamanho médio dos frutos de $M$. glaucescens $(1,23 \mathrm{~cm}$; $\mathrm{n}=20)$ e $M$. paucispinus $(1,58 \mathrm{~cm} ; \mathrm{n}=20)$ definimos como referência o valor de $0,3 \mathrm{~cm}$ de comprimento do fruto para classificar os frutos como não-disponíveis e disponíveis. $\mathrm{O}$ critério adotado independe do tamanho do fruto, conferindo maior segurança no dado registrado, uma vez que não é possível determinar com exatidão quanto do comprimento do fruto ainda se encontra dentro do cefálio sem arrancá-lo.

Durante a investigação do padrão diário de liberação dos frutos e remoção por lagartos foram estimadas a temperatura média dos cefálios e a temperatura ambiente, de hora em hora, das $06 \mathrm{~h} 00$ às $18 \mathrm{~h} 00$. A temperatura do cefálio foi medida com termômetro digital de resposta rápida com ponta que faz leitura de superfície de contato, a qual foi inserida 1 $\mathrm{cm}$ no cefálio no lado oposto a incidência solar para evitar que a radiação interferisse nos dados; quando havia frutos emergido ou estrugido no lado oposto a incidência solar, a ponta do termômetro foi inserida a $2 \mathrm{~cm}$ do fruto para evitar que a pressão exercida pela mesma promovesse ou acelerasse a liberação. A temperatura ambiente foi medida com um termo-higrômetro digital, fixado a $1 \mathrm{~m}$ do solo ao abrigo do sol. Utilizou-se o coeficiente de correlação de 
Pearson para verificar a correlação entre: a taxa de extrusão e a taxa de remoção (utilizando-se um time-lag + 1); a taxa de remoção e a temperatura ambiente e as taxas de emergência e extrusão e a variação térmica do cefálio.

Para detectar o padrão de distribuição espacial das duas espécies foi utilizada uma grade com 400 parcelas de $2 \times 2 \mathrm{~m}\left(4 \mathrm{~m}^{2}\right)$, que cobriu uma área total de $1600 \mathrm{~m}^{2}$. Em cada unidade amostral (parcela) foi registrado o número de indivíduos adultos (com cefálio) e jovens (sem cefálio), sendo que os indivíduos que ficaram sob a linha superior e direita das parcelas foram incluídos na amostra, enquanto os indivíduos sob a linha inferior e esquerda foram excluídos. A distribuição espacial foi avaliada através do coeficiente de dispersão $(\mathrm{CD})$, definido pela razão entre a variância e a média do número de indivíduos nas parcelas. Quando $\mathrm{CD}$ = 1, o padrão de distribuição é aleatório; quando $\mathrm{CD}<1$, a distribuição é regular e quando $C D>1$, a distribuição é agregada (Brower \& Zar 1984).

\section{Resultados}

\section{Dispersão de sementes}

Duas espécies de lagartos, Cnemidophorus ocellifer (Teiidae) e Tropidurus cocorobensis (Tropiduridae), foram observadas consumindo os frutos de M. glaucescens e $M$. paucispinus (Fig. 1) e uma terceira espécie de lagarto, Tropidurus semitaeniatus (Tropiduridae), foi vista consumindo os frutos de M. glaucescens fora da área de estudo. O consumo dos frutos por estes frugívoros foi mais intenso entre $09 \mathrm{~h} 00$ e 14 h00 (Fig. 2, 3).

Com relação ao comportamento de forrageio, observou-se que C. ocellifer pegava os frutos que se encontravam apenas sobre o solo, enquanto T. cocorobensis consumia os frutos que se encontravam sobre o solo, cladódio e cefálio. Estes lagartos subiam nos cactos rapidamente até atingir os frutos ou pulavam em cima do cefálio, sendo que em alguns casos, um único lagarto exibia várias posições para pegar um fruto de difícil acesso, chegando, às vezes a contorcer o próprio corpo. Tropidurus cocorobensis consumiu os frutos tanto de M. glaucescens como de M. paucispinus mais freqüentemente que C. ocellifer (Tab. 1).

Com relação ao tratamento dado aos diásporos, $C$. ocellifer foi observado consumindo os frutos parcialmente, por completo ou apenas os macerando com a boca e os abandonando em seguida, enquanto os indivíduos de $T$. cocorobensis consumiram os frutos por completo (Tab. 1). O fato de se encontrar frutos mordidos no cefálio indica que esta última espécie também consome os frutos parcialmente. Os frutos consumidos por completo eram abocanhados e ingeridos após alguns movimentos com a mandíbula. Em alguns casos, o fruto era mordido inicialmente e o resto que pendia consumido em seguida.

Os lagartos foram freqüentemente observados nas bordas das moitas, margeando a vegetação, sobre o folhedo; quando iam apanhar os frutos agiam rapidamente e os transportavam na boca por curtas distâncias para áreas "protegidas" por vegetação rala, onde os consumiam. Em alguns casos, os frutos foram ingeridos nos locais onde foram apanhados, inclusive em cima do cefálio pela espécie de Tropidurus. Esta espécie foi observada também derrubando os frutos no chão e depois os consumindo e/ou transportando-os para tais áreas mais protegidas.

Em cativeiro, os lagartos defecaram sementes intactas geralmente após um dia do consumo. O número de sementes por fezes foi variado, sendo algumas vezes próximo da média de sementes encontradas num fruto. Em alguns casos, os lagartos defecaram o "fruto inteiro", i.e., o epicarpo do fruto rompido, translúcido, envolvendo algumas sementes.

As taxas de germinação das sementes de M. glaucescens e $M$. paucispinus provenientes das fezes e de seus respectivos controle (Tab. 2) não diferiram significativamente, sugerindo que a passagem das sementes pelo trato digestivo dos lagartos não estimula ou inibe o processo de germinação das sementes dessas duas espécies de Melocactus.

A espécie de formiga Ectatomma edentatum (Ponerinae, Ectatommini) foi observada dispersando as sementes de M. glaucescens e M. paucispinus e duas outras espécies, Acromyrmex laticeps nigrosetosus (Myrmicinae, Attini) e Solenopsis substituta (Myrmicinae, Solenopsidini), dispersando as sementes de M. paucispinus (Fig. 1). Uma quarta espécie, Dorymyrmex thoracicus (Dolichoderinae, Dolichoderini), foi observada extraindo pequenas porções (ca. 0,1 $\mathrm{cm}$ ) dos frutos de M. paucispinus e possivelmente líquidos, porém não removeu sementes.

Ectatoma edentatum foi vista explorando o campo individualmente ou em número reduzido por área (duas a três operárias); o número de formigas encontrado por diásporo foi sempre um, assim como por indivíduo. As demais espécies de formigas, como esperado devido ao seu sistema de coleta por cooperação, foram encontradas em número mais elevado explorando os diásporos e/ou indivíduos de M. paucispinus (Tab. 1). A atividade de consumo dos frutos pelas formigas nos dias ensolarados foi maior antes das $09 \mathrm{~h} 00$ e a partir das $17 \mathrm{~h} 00$, sendo ausente em horários mais quentes do dia. No total foram observadas 14 formigas transportando as sementes das espécies de Melocactus em estudo, as quais abandonaram as sementes antes de chegarem aos ninhos. A distância da dispersão variou de 0,5 a 7 $\mathrm{m}$ (média $\pm \mathrm{EPM}=4 \pm 0,58$ ).

\section{Padrão diário de liberação dos frutos e remoção por lagartos}

Vinte e quatro indivíduos de M. glaucescens produziram frutos no primeiro dia de observação e 16 no segundo. Oito indivíduos de M. paucispinus produziram no primeiro dia, 15 no segundo e 17 no terceiro. As taxas de emergências, extrusão e remoção de frutos foram similares entre o primeiro e o segundo dia de observação em M. glaucescens, cujas temperaturas médias ambientais foram de $23,7^{\circ} \mathrm{C}$ (18,7 mín. - 27máx.) e 22,7 ${ }^{\circ} \mathrm{C}$ (19mín. - 


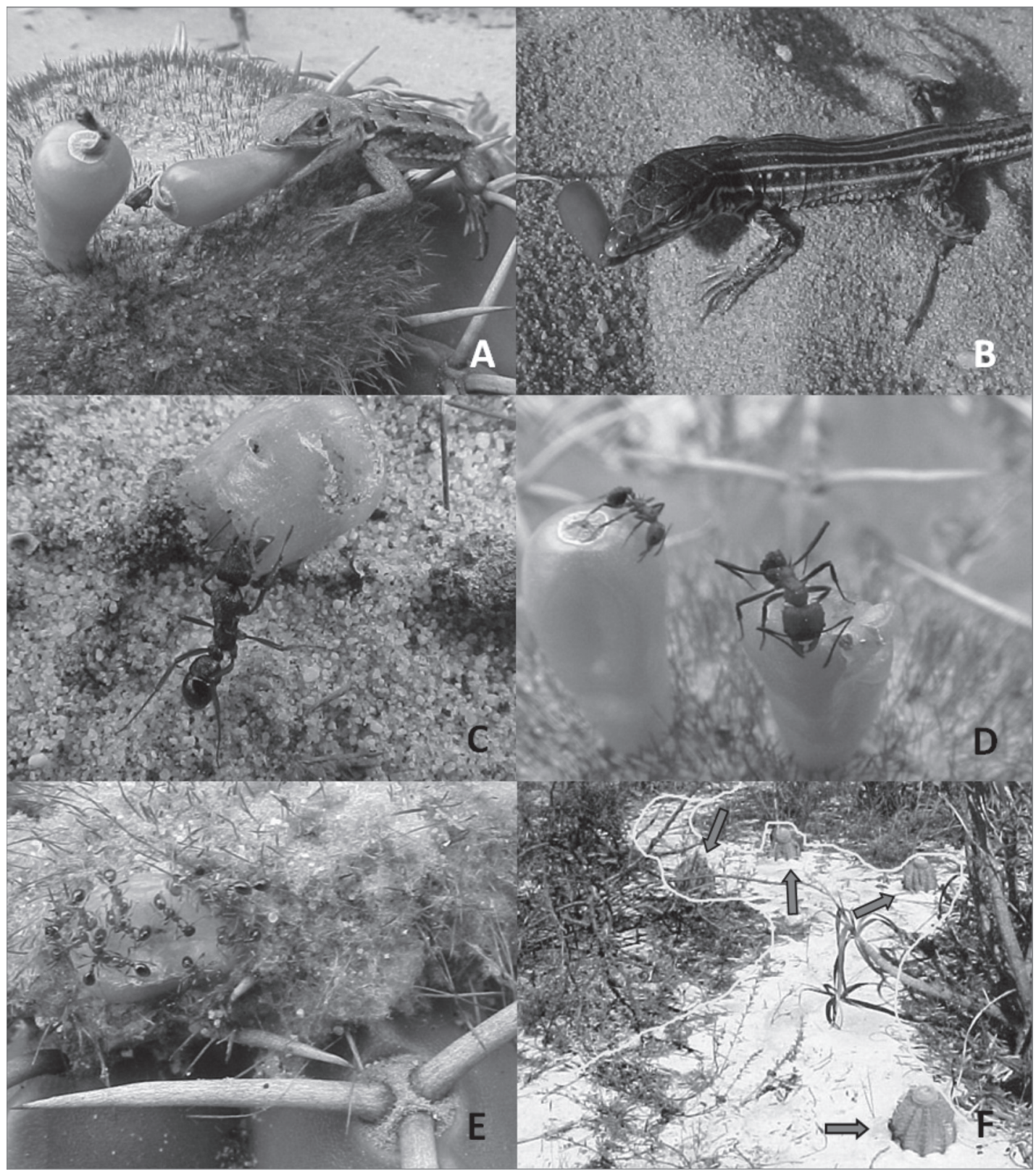

Figura 1. Tropidurus cocorobensis consumindo o fruto de Melocactus paucispinus (A); Cnemidophorus ocellifer consumindo o fruto de M. glaucescens (B); Ectatomma edentatum transportando o fruto de M. paucispinus (C); Acromyrmex laticeps nigrosetosus (D) e Solenopsis substituta (E) explorando o fruto de M. paucispinus. Indivíduos de M. glaucescens margeando a vegetação (F). Morro do Chapéu, Bahia.

26,9máx.), respectivamente (Fig. 2). Em M. paucispinus, as taxas de emergência e extrusão foram menores no primeiro dia de observação, cuja temperatura média foi de $25,5^{\circ} \mathrm{C}$ (18,5mín. - 29máx.) e similares entre o segundo e terceiro dia, com temperaturas médias de $23,8^{\circ} \mathrm{C}(17,5$ mín. 27,5máx.) e 19,8 ${ }^{\circ} \mathrm{C}$ (15mín. - 24máx.), respectivamente. A taxa de remoção foi menor no terceiro dia, de temperatura mais amena (Fig. 3).

Entre o primeiro e o segundo dia de observação em M. glaucescens (período das $18 \mathrm{~h} 00$ às $06 \mathrm{~h} 00$ ) ocorreu à emergência e a extrusão de três frutos. Entre o segundo e o terceiro dia de observação em $M$. paucispinus (dias observados continuadamente) foi verificada, por sua vez, a emergência de cinco frutos e a extrusão de um.

Por intervalo-hora, as taxas de emergência e extrusão de frutos variaram, respectivamente, de 0 a 7 e 0 a 8 em $M$. glaucescens e de 0 a 5 e 0 a 4 em M. paucispinus. O número de frutos disponíveis por intervalo-hora variou de 4 a 29 para M. glaucescens e de 1 a 9 para M. paucispinus. A taxa de remoção variou de 0 a 1 em $M$. paucispinus e de 0 a 0,29 em $M$. glaucescens. O número de frutos removidos variou de 0 a 8 frutos em $M$. glaucescens e de 0 a 5 em M. paucispinus.

De um modo geral, a taxa de emergência foi mais elevada no início da manhã, diminuindo ao longo do dia. A taxa de 

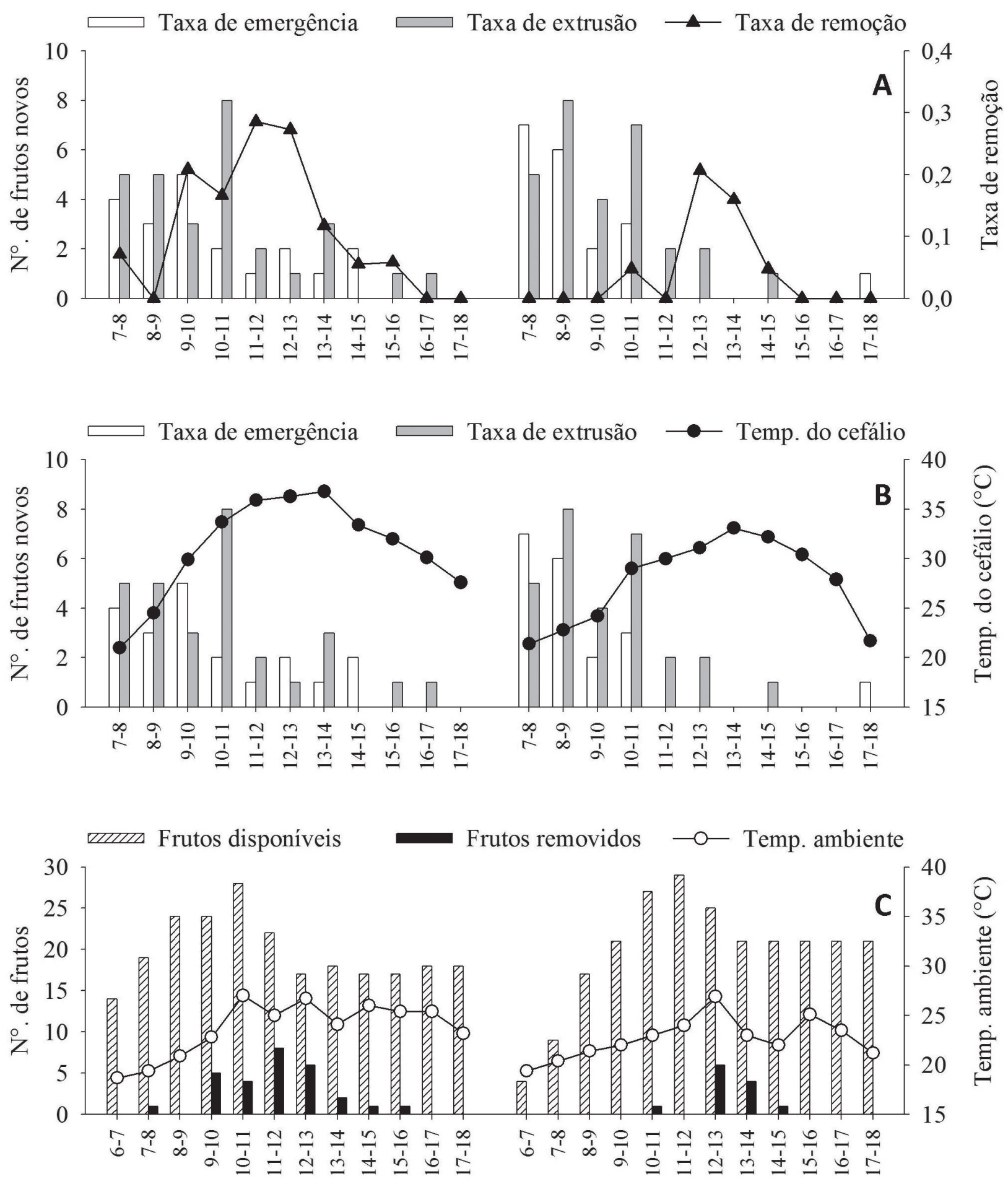

Primeiro dia

Segundo dia

Figura 2. Taxas de emergência, extrusão e remoção de frutos de Melocactus glaucescens (A); taxas de emergência e extrusão de frutos e temperatura média do cefálio (B); frutos disponíveis e removidos por lagartos e temperatura ambiente (C) ao longo de dois dias. Morro do Chapéu, Bahia, junho de 2003. 

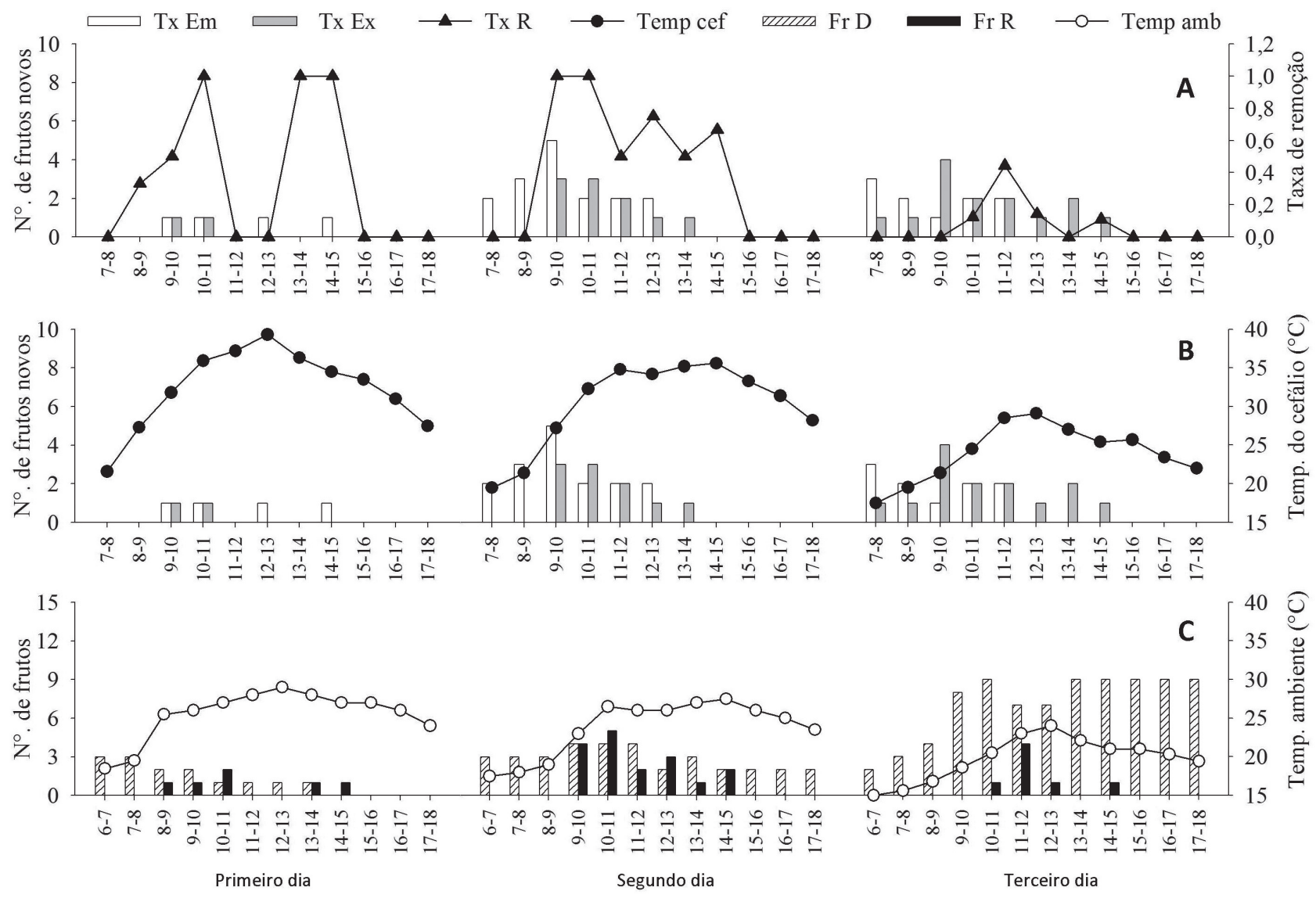

Figura 3. Taxas de emergência (Tx Em), extrusão (Tx Ex) e remoção (Tx R) de frutos de Melocactus paucispinus (A); taxas de emergência (Tx Em) e extrusão (Tx Ex) de frutos e temperatura média do cefálio (Temp cef) (B); frutos disponíveis (Fr D) e removidos (Fr R) por lagartos e temperatura ambiente (Temp amb) (C) ao longo de três dias. Morro do Chapéu, Bahia, junho de 2003.

extrusão foi mais intensa do meio para o final da manhã, sendo consideravelmente menor à tarde. A taxa de remoção foi concentrada nas horas centrais do dia, com declínio no primeiro e segundo dia de observação para M. paucispinus em torno do meio dia (Fig. 2, 3).

Em geral, o pico de expulsão dos frutos coincidiu com os picos de remoção (considerando um time-lag +1 ) ou antecedeu a estes (Fig. 2, 3). A taxa de extrusão de frutos e a taxa de remoção não se correlacionaram significativamente em M. glaucescens $(\mathrm{r}=0,17 ; \mathrm{p}>0,05 ; \mathrm{n}=20)$ e em $M$. paucispinus $(\mathrm{r}=0,18, \mathrm{p}>0,05 ; \mathrm{n}=30)$. A taxa de remoção foi diretamente correlacionada com a temperatura ambiente, tanto em M. glaucescens $(\mathrm{r}=0,50 ; \mathrm{p}<0,05 ; \mathrm{n}=22)$, como em M. paucispinus ( $\mathrm{r}=0,50, \mathrm{p}<0,05, \mathrm{n}=33)$.

\section{Padrão de liberação dos frutos e variação térmica do cefálio}

A menor temperatura do cefálio de $M$. glaucescens e M. paucispinus registrada foi de $19,4^{\circ} \mathrm{C}$ e $16,5^{\circ} \mathrm{C}$, respectivamente, quando a temperatura ambiente encontrava-se em $18,7^{\circ} \mathrm{C}$ e $15^{\circ} \mathrm{C}$, respectivamente. A maior temperatura registrada, por sua vez, foi de $36,8{ }^{\circ} \mathrm{C}$ e $39,3{ }^{\circ} \mathrm{C}$ para $M$. glaucescens e $M$. paucispinus, respectivamente, quando a temperatura ambiente encontrava-se em $24,1^{\circ} \mathrm{C}$ e $29,0{ }^{\circ} \mathrm{C}$, respectivamente. A temperatura do cefálio de ambas as espécies de Melocactus, no lado oposto à incidência solar, foi em torno de $2{ }^{\circ} \mathrm{C}$ mais baixa $(\mathrm{n}=10)$ que a temperatura do lado em que os raios solares incidem diretamente. Medidas não sistemáticas, em dias ensolarados, revelaram que a temperatura do cefálio pode chegar a $46^{\circ} \mathrm{C}$, quando a temperatura ambiente encontrava-se em torno de $33^{\circ} \mathrm{C}$.

Em termos gerais, a temperatura média do cefálio aumentou gradativamente ao longo do dia e começou a diminuir do meio para o final da tarde (Fig. 2, 3). A taxa de emergência dos frutos de $M$. glaucescens correlacionou negativamente com a temperatura média do cefálio em um dos dias em que foram realizadas as investigações e na análise de toda a amostra (dados dos dois dias). A taxa de extrusão não correlacionou significativamente, considerando tanto os registros diários individualmente como toda a amostra (Tab. 3). As taxas de emergência e extrusão dos frutos de $M$. paucispinus não correlacionaram significativamente com a temperatura média do cefálio, considerando tanto 
Tabela 1. Freqüência (Fr.) de consumo dos frutos de Melocactus glaucescens e M. paucispinus por lagartos e de interações diásporos-formigas. Tipo de tratamento dado aos diásporos pelos lagartos (CT - consumo total do fruto, CP - consumo parcial, M - maceração do fruto com a boca e abandono) e pelas formigas [TG transporte de porções grandes de fruto $( \pm 0,7 \mathrm{~cm}), \mathrm{TM}$ - transporte de porções médias $( \pm 0,3 \mathrm{~cm})$, TP - transporte de porções pequenas $( \pm 0,1 \mathrm{~cm})$, TS - transporte de sementes]. Número de formigas por diásporo/indivíduo de Melocactus (No. formigas). Morro do Chapéu, Bahia, $2002-2003$.

\begin{tabular}{|c|c|c|c|c|c|c|}
\hline & \multicolumn{3}{|c|}{ M. glaucescens } & \multicolumn{3}{|c|}{ M. paucispinus } \\
\hline & Fr. & Tratamento & No. formigas & Fr. & Tratamento & No. formigas \\
\hline \multicolumn{7}{|l|}{ Lagartos } \\
\hline Tropidurus cocorobensis & 9 & $\mathrm{CT}$ & & 11 & СТ & \\
\hline Cnemidophorus ocellifer & 3 & CT, CP, M & & 4 & CT, M & \\
\hline \multicolumn{7}{|l|}{ Formigas } \\
\hline Ectatomma edentatum & 5 & TG & $1 / 1$ & 7 & TG & $1 / 1$ \\
\hline Acromyrmex laticeps nigrosetosus & 0 & & & 10 & TM, TS & $1-5 / 1-20$ \\
\hline Solenopsis substituta & 0 & & & 8 & TP, TS & $1-15 / 5-30$ \\
\hline Dorymyrmex thoracicus & 0 & & & 3 & TP & $1-10 / 6-22$ \\
\hline
\end{tabular}

Tabela 2. Taxas de germinação (\%) das sementes de Melocactus glaucescens e M. paucispinus provenientes das fezes de lagartos e diretamente da planta mãe (controle). Ver métodos para detalhes dos tratamentos. Morro do Chapéu, Bahia, 2003.

\begin{tabular}{|c|c|c|c|c|}
\hline & M. glaucescens & Controle & M. paucispinus & Controle \\
\hline Tropidurus cocorobensis & $6 \pm 1,2$ & \multirow{2}{*}{$13 \pm 6,4$} & $83 \pm 4,7$ & $94 \pm 2,6$ \\
\hline Cnemidophorus ocellifer & $13 \pm 6,4$ & & $8 \pm 1,6$ & $31 \pm 13,3$ \\
\hline
\end{tabular}

os registros diários individualmente como toda a amostra (dados dos três dias), (Tab. 3).

\section{Distribuição espacial}

$\mathrm{Na}$ área amostrada foi registrado um total de 58 indivíduos de M. glaucescens, sendo 29 adultos (com cefálio) e 29 jovens (sem cefálio). Para M. paucispinus, foram registrados 250 indivíduos; destes 77 eram adultos e 173 eram jovens. Também foram registrados 5 e 14 indivíduos mortos de M. glaucescens e M. paucispinus, respectivamente, na área amostral considerada. O coeficiente de dispersão de $M$. glaucescens foi 1,48 e o de M. paucispinus foi 1,62, o que caracteriza o padrão de distribuição espacial dos indivíduos de ambas as populações como agregado. Os indivíduos foram freqüentemente observados em espaços abertos da vegetação, constituindo pequenos agrupamentos e/ou margeando a periferia das moitas (Fig. 1F).

\section{Discussão}

\section{Dispersão de sementes}

Os lagartos Tropidurus cocorobensis e Cnemidophorus ocellifer e as formigas Ectatomma edentatum, Acromyrmex laticeps nigrosetosus e Solenopsis substituta atuam como dispersores locais de sementes de M. glaucescens e M. paucispinus nas áreas estudadas, com destaque para T. cocorobensis. O comportamento de forrageio, a frequência e o tratamento dado aos frutos por $C$. ocellifer indicam que esta espécie de lagarto é menos eficiente como dispersora das sementes de $M$. glaucescens e M. paucispinus que T. cocorobensis. Tropidurus cocorobensis busca ativamente os frutos de Melocactus e os consome com maior frequência, geralmente por completo. O consumo de material vegetal por espécies de Tropidurus tem sido amplamente reportado (Araujo 1984, Fialho 1990, Figueira et al. 1994, Rocha \& Bergallo 1994, Teixeira \& Giovanelli 1999), enquanto há poucos registros para C. ocellifer (Araujo 1984). É possível que C. ocellifer macere parte dos frutos de Melocactus que encontre, abandonando-os em seguida, com o intuito de obter água, porém a polpa pegajosa dos frutos durante esta ação pode fazer algumas sementes ficarem aderidas na boca destes lagartos e então serem dispersas. Em geral, as maiores taxas de remoção dos frutos pelos lagartos situaram-se dentro da faixa de atividade horária esperada para estes vertebrados, animais pecilotérmicos (heliotérmicos em sua maioria), cujas atividades são condicionadas pela temperatura ambiental (Araujo 1984, Vitt et al. 1996, Mesquita \& Colli 2003).

As sementes de M. glaucescens e M. paucispinus não perderam a dormência ao passar pelo trato digestivo dos lagartos, porém se mantiveram intactas, confirmado o status de dispersoras destas espécies de lagartos. De forma similar 
Tabela 3. Coeficiente de correlação de Pearson entre as taxas de emergência (Tx Emer) e extrusão (Tx Extr) de frutos e a temperatura média diária dos cefálios de dois e três dias, respectivamente, para M. glaucescens e M. paucispinus. Morro do Chapéu, Bahia, junho de 2003.

\begin{tabular}{|c|c|c|c|c|c|}
\hline & & \multirow{2}{*}{\multicolumn{2}{|c|}{ M. glaucescens }} & & \\
\hline & & & & \multicolumn{2}{|c|}{ M. paucispinus } \\
\hline & & Tx Emer & Tx Extr & Tx Emer & Tx Extr \\
\hline \multirow{4}{*}{ Temperatura cefálio } & $1^{\circ} \mathrm{dia}$ & $-0,40$ & $-0,25$ & 0,46 & 0,14 \\
\hline & $2^{\circ}$ dia & $-0,73^{*}$ & $-0,45$ & $-0,47$ & 0,22 \\
\hline & $3^{\circ}$ dia & & & $-0,51$ & 0,03 \\
\hline & Amostra total & $-0,52^{*}$ & $-0,33$ & $-0,27$ & $-0,14$ \\
\hline
\end{tabular}

* significativo em nível de $5 \%$

ao presente trabalho, outros estudos têm reportado a ausência de efeito do trato digestivo de répteis sobre a germinação de sementes (Valido \& Nogales 1994, Castilla 2000, Traveset \& Verdú 2002), embora outros têm registrado um aumento na germinação (Cobo \& Andreu 1988, Figueira et al. 1994, Valido \& Nogales 1994).

As taxas de germinação das sementes de M. glaucescens (tratamentos e controle) foram baixas, porém compatíveis com a idade pós-colheita das sementes utilizadas (44dias). A taxa de germinação das sementes de $M$. glaucescens começa a aumentar após 134 dias de idade pós-colheita, sendo significativamente maior com 308 dias (Fonseca 2004). Já a diferença entre as taxas de germinação das sementes de $M$. paucispinus provenientes das fezes de $T$. cocorobensis e C. ocellifer, deve-se às condições dos lotes testados, obtidos em fevereiro (verão) e junho (inverno) de 2003, respectivamente. As sementes de M. paucispinus produzidas em junho de 2003 apresentaram dormência (Fonseca 2004), que pode estar associada ao período em que os frutos foram desenvolvidos e maturados (Fenner 1991, Gutterman 2000).

A importância de Ectatoma edentatum e de espécies da tribo Attini na dispersão de sementes de plantas tropicais tem sido documentada (Ferreira 1998, Leal \& Oliveira 1998). Solenopsis substituta e Dorymyrmex thoracicus são mais oportunistas (onívoras); coletam principalmente pequenos insetos ou cadáveres de insetos, mais não rejeitam outros substratos principalmente quando tem carboidratos a serem coletados (J. H. C. Delabie, comunic. pess.).

Ectatoma edentatum, por ser maior que A. laticeps nigrosetosus e S. substituta, pode transportar individualmente um maior número de sementes (aderidas a porções grandes de frutos), porém as últimas, podem transportar coletivamente um maior número, devido ao sistema de coleta por cooperação que apresentam. Este sistema é vantajoso quando as fontes de alimento estão concentradas, enquanto a coleta individual é vantajosa quando os recursos estão dispersos (Caetano et al. 2002). A atuação das três formigas na dispersão de $M$. paucispinus contempla ambas as situações, o que contribui para tornar a mirmecocoria importante nesta população.
Ectatoma edentatum e A. laticeps nigrosetosus apresentam mandíbulas desenvolvidas capazes de cortar a epiderme delicada dos frutos das espécies de Melocactus e remover porções, enquanto S. substituta e D. thoracicus parecem ser atraídas e forragear apenas frutos já rompidos (Taylor 1991, 2000). Embora não investigamos o tratamento dado às sementes pelas formigas nos ninhos, o fato das formigas transportarem (com exceção de $D$. thoracicus) e abandonarem as sementes de $M$. glaucescens e $M$. paucispinus em um determinado momento indica que ao menos uma parte das sementes removidas por estas é realmente dispersa. As distâncias de dispersão observadas pelo presente estudo encontram-se dentro da faixa de amplitude já relatada para as Ponerinae e Myrmicinae. Gómez \& Espadaler (1998), em uma revisão sobre distância de dispersão por formigas, encontrou para as Ponerinae uma média de $1,41 \mathrm{~m}(\mathrm{n}=412)$ e amplitude de $0,01-20,5 \mathrm{~m}$, e para as Myrmicinae, média de 0,69 m $(\mathrm{n}=1349)$ e amplitude de 0,01-16,97 m.

\section{Padrão de liberação dos frutos e remoção por lagartos}

Maiores taxas de emergência de frutos no início da manhã e de extrusão do meio para o final da manhã, diminuindo ao longo do dia, como aqui observado, foi anteriormente registrado em Melocactus violaceus (Figueira et al. 1994). Porém, diferente daquele estudo, não encontramos correlação entre a taxa de extrusão dos frutos e a taxa de remoção com time-lag +1 , assim como, uma tendência bimodal na taxa de extrusão dos frutos, de forma similar ao modelo de atividade horária de T. torquatus registrado por Araujo (1984).

$\mathrm{O}$ aumento na taxa de extrusão de frutos seguido de um declínio e levemente de um aumento e um declínio, que pode ser visualizado ligeiramente em alguns dias observados, não representa uma tendência bimodal, similar à atividade de T. torquatus. O modelo bimodal de atividade horária encontrado para T. torquatus por Araujo (1984), apresenta uma redução de atividade nas horas centrais do dia, quando a temperatura se encontra mais elevada, o que não ocorreu com a taxa de extrusão 
dos frutos de M. glaucescens e M. paucispinus. O fato das taxas de emergência e extrusão serem intensas durante a manhã e consideravelmente reduzidas à tarde aumenta as chances dos frutos serem removidos pelos lagartos ao longo do mesmo dia em que foram liberados, evitando dissecação e predação, podendo ser um ajuste ecológico destas espécies de Melocactus.

A correlação positiva encontrada entre as taxas de remoção e a temperatura ambiente está relacionada ao fato dos lagartos terem suas atividades influenciadas por este fator ambiental (Araujo 1984, Vitt et al. 1996, Mesquita \& Colli 2003). Este fato, também pode explicar a diminuição da taxa de remoção no terceiro dia de observação de M. paucispinus, de temperatura mais amena, bem como a disponibilidade de frutos ao longo do dia.

\section{Padrão de liberação dos frutos e variação térmica do cefálio}

Os frutos de Melocactus glaucescens e M. paucispinus foram registrados emergindo e estrugindo tanto em temperaturas baixas, do início da manhã, como em temperaturas mais elevadas. A emergência e a extrusão de frutos à noite indicam que os frutos podem ser liberados em temperaturas mais baixas que as registradas. No entanto, a taxa de emergência e extrusão desse período é baixa quando comparada ao período diurno. Mesmo considerando possíveis remoções noturnas, o número de frutos disponíveis no final do dia, após atuação dos lagartos, foi bem maior durante três dos cinco dias observados. Similarmente, a emergência de frutos em $M$. violaceus foi baixa durante a noite, com frutos emergindo, aproximadamente, três vezes mais durante o dia, e se tornando disponíveis seis vezes mais (Figueira et al. 1994).

Embora seja possível que a temperatura seja responsável por esta diferença de intensidade, como sugerido por Figueira et al. (1994), não encontramos correlação positiva desta com a emergência e a extrusão dos frutos nas diferentes situações analisadas. A correlação negativa com a taxa de emergência, encontrada em algumas situações, é reflexo desta ter sido em geral mais elevada no início da manhã, diminuindo com o progredir do dia. Além disso, I. G. Varassin (dados não public.) analisou a temperatura do cefálio de indivíduos de M. violaceus em frutificação, na Reserva Florestal de Linhares, município de Linhares - ES, e não observou nenhum padrão entre a temperatura do cefálio e a extrusão de frutos, que se iniciou desde temperaturas menores, continuando ao longo do dia. Essa autora concluiu que a emergência é condicionada ao tempo de maturação do fruto e que o processo de expulsão pode ser desencadeado com o início do aquecimento do cefálio e se estender durante o dia todo, não sendo necessárias temperaturas mais elevadas para o início do processo.

Dos 58 frutos de M. glaucescens e 26 de M. paucispinus que foram registrados estrugidos, 46 e 23 frutos, respectiva- mente, foram registrados em horário(os) anterior(es) como frutos não disponíveis. Sendo assim, a tendência encontrada para uma maior concentração da taxa de extrusão do meio para o final da manhã pode ser conseqüência do processo natural de expulsão do fruto, iniciado em temperaturas menores. $\mathrm{O}$ fato das taxas de emergência e extrusão de $M$. paucispinus terem sido menores no primeiro dia (dia mais quente), por outro lado, sugere que existe limiar máximo de temperatura favorável. A diminuição da extrusão nas horas centrais do dia (caracterizando o padrão como bimodal), verificada por Figueira et al. (1994), também corrobora com esta idéia.

Os frutos de M. glaucescens e M. paucispinus se encontram dentro do cefálio presos na base por um tecido extremamente delicado que rompe quando o fruto torna-se maduro (Fonseca 2004). A própria pressão criada com o aumento do tamanho do fruto durante seu desenvolvimento gera uma pressão interna que pode impulsioná-lo até certo ponto ou totalmente . Esta pressão é potencializada pela arquitetura do cefálio, visto que as cerdas possuem uma tensão na base, devido sua forte junção. Além disto, os frutos internamente se desenvolvem muito próximos um dos outros (Fonseca 2004), de forma que a maturação e a saída de um novo fruto podem empurrar um fruto que não saiu totalmente, ou até mesmo restabelecer a pressão em torno deste (fruto imóvel), levando a sua expulsão completa.

A diminuição da extrusão à tarde pode estar relacionada ao fato de que a maioria dos frutos maduros já tenha sido liberada pela manhã e/ou pelo fato das fibras do cefálio já terem atingido o máximo de dilatação.

\section{Padrão de distribuição espacial}

A distribuição espacial de uma população é dependente do padrão de dispersão das sementes e da probabilidade de sobrevivência das plântulas, a qual pode ser afetada por fatores abióticos ou bióticos, que constituem o mosaico de condições ambientais existentes na área (Hubbell 1980, Oliveira et al. 1989).

A dispersão a curta distância promovida pelos lagartos territorialistas observados e o comportamento (supostamente de defesa ou de termo-regulação da temperatura) de forragear na periferia da vegetação, bem como apanhar os frutos rapidamente e os consumir geralmente em locais protegidos por vegetação rala, favorece a distribuição agregada das sementes, freqüentemente às margens da vegetação, onde muitas plântulas encontram locais propícios e seguros para o recrutamento (Ellner \& Shmida 1981, Hughes 2009, Hughes et al. 2011).

As formigas, em sua maioria, abandonaram as sementes em locais arenosos, sem ou com escassa vegetação, inclusive embaixo de folhedo, onde as micro-condições mais apropriadas de umidade, temperatura e nutrientes favorece a germinação das sementes (Horvitz 1981).

Diante do exposto, concluímos que tanto os lagartos como as formigas figuram como dispersores locais de $M$. 
glaucescens e M. paucispinus, influenciando o arranjo espacial dos indivíduos, que tendem a ofertar frutos em horários favoráveis a dispersão, como resultado da combinação da estrutura florífera e temperatura.

\section{Agradecimentos}

Os autores agradecem a Rubeney Fonseca e a Delmar Alvim, pela ajuda nos trabalhos de campo; à Roy Funch, pela ajuda com o abstract; e aos revisores anônimos, pelas valiosas críticas e sugestões. Este trabalho foi financiado pelo Fundo Nacional de Meio Ambiente (FNMA \#75/2001). R. B. S. Fonseca recebeu bolsa de estudos da CAPES (Coordenação de Aperfeiçoamento de Pessoal de Nível Superior).

\section{Referências bibliográficas}

Araujo, A.F.B. 1984. Padrões de divisão de recursos em uma comunidade de lagartos de restinga. Pp. 327-342. In: Lacerda, L.D.; Araujo, D.S.D.; Cerqueira, R. \& Turcq, B. (Eds.). Restingas: origens, estrutura, processos. Niterói, CEUFF.

Augspurger, C.K. 1986. Morphology and dispersal potential of winddispersed diaspores of neotropical trees. American Journal of Botany 73: 353-363.

Bregman, R. 1988. Forms of seed dispersal in Cactaceae. Acta Botanica Neerlandesa 37: 395-402.

Brower, J.E. \& Zar, J.H. 1984. Field \& laboratory methods for general ecology. 2 ed. Wm. C. Brown Publishers, Dubuque, Iowa.

Caetano, F.H.; Jaffé, K. \& Zara, F. J. 2002. Formigas: biologia e anatomia. Rio Claro, F.H.C.

Castilla, A.M. 2000. Does passage time through the lizard Podarcis lilfordi's guts affect germination performance in the plant Withania frutescens. Acta Oecologica 21: 119-124.

Chapman, C.A. 1989. Primate seed dispersal: the fate of dispersed seeds. Biotropica 21: 148-154.

Cobo, M. \& Andreu, A.C. 1988. Seed consumption and dispersal by the spur-thighed tortoise Testudo graeca. Oikos 51: 267-273.

CPRM - Companhia de Pesquisa de Recursos Minerais. 1995. Projetos mapas municipais município de Morro do Chapéu (BA). Salvador.

Dirzo, R. \& Dominguez, C.A. 1986. Seed shadows, seed predation and the advantages of dispersal. Pp. 237-249. In: Estrada, A. \& Fleming, T.H. (Eds.). Frugivores and seed dispersal. Dordrecht, Dr. W. Junk Publishers.

Ellner, S. \& Shmida, A. 1981. Why are adaptations for long-range seed dispersal rare in desert plants? Oecologia 51: 133-114.

Fenner, M. 1991. The effects of the parent environment on seed germinability. Seed Science Research 1: 75-84.

Ferreira, M.A.P. 1998. Interação formiga-planta em solo de mata atlântica: influência das formigas na ecologia de frutos e sementes não-mirmecocóricas. Tese de Doutorado, Universidade Estadual de Campinas, Campinas.

Fialho, R.F. 1990. Seed dispersal by a lizard and a treefrog - Effect of dispersal site on seed survivorship. Biotropica 22: 423-424.

Figueira, J.E.C.; Vasconcellos-Neto, J.; Garcia, M.A. \& Souza, A.L.T. 1994. Saurocory in Melocactus violaceus (Cactaceae). Biotropica 22: 295-301.

Fonseca, R.B.S. 2004. Fenologia reprodutiva e dispersão de Melocactus glaucescens Buining \& Brederoo e M. paucispinus G. Heimen \& R. Paul (Cactaceae) no Município de Morro do Chapéu, Chapada Diamantina - Bahia - Brasil. Dissertação de Mestrado, Universidade Estadual de Feira de Santana, Feira de Santana.
Gómez, C. \& Espadaler, X. 1998. Myrmecochorous dispersal distances: a world survey. Journal of Biogeography 25: 573-580.

Gutterman, Y. 2000. Maternal effects on seeds during development. Pp. 59-84. In: Fenner, M. (Ed.). Seeds: The Ecology of Regeneration in Plant Communities, 2nd edition. (C) CAB International.

Horvitz, C.C. 1981. Analysis of how ant behaviours affect germination in a tropical myrmecochore Calathea microcephala (P. \& E.) Koernicke (Marantaceae): microsite selection and aril removal by neotropical ants, Odontomachus, Pachycondyla, and Solenopsis (Formicidae). Oecologia 51: 47-52.

Howe, H.F. 1989. Scatter-and clump-dispersal and seedling demography: hypothesis and implications. Oecologia 79: 417-426.

Hubbell, S.P. 1980. Seed predation and coexistence of tree species in tropical forests. Oikos 35: 214-229.

Hughes, F.M. 2009. Ecologia reprodutiva e morfometria de Melocactus ernestii (Vaupel) no município de Nova Itarana. Dissertação de mestrado, Universidade Estadual de Feira de Santana, Feira de Santana.

Hughes, F.M.; De La Cruz Rot, M.; Romão, R.L. \& Castro, M.S. 2011. Dinâmica espaço-temporal de Melocactus ernestii subsp. ernestii (Cactaceae) no Nordeste do Brasil. Revista Brasileira de Botânica 34: 389-402.

Jordano, P. 1987. Avian fruit removal: effects of fruit variation, crop size, and insect damage. Ecology 68: 1711-1723.

Leal, I.R. \& Oliveira, P.S. 1998. Interactions between fungus-growing ants (Attini), fruits and seeds in cerrado vegetation in southeast Brazil. Biotropica 30: 170-178.

Mesquita, D.O.E Colli, G.R. 2003. The ecology of Cnemidophorus ocellifer (Squamata, Teiidae) in a neotropical savanna. Journal of Herpetology 37: 498-509.

Oliveira, P.E.A.M. \& Moreira, A.G. 1992. Anemocoria em espécies de cerrado e mata de galeria de Brasília, DF. Revista Brasileira de Botânica 15: 163-164.

Oliveira, P.E.A.M.; Ribeiro, J.F. \& Gonzales, M.I. 1989. Estrutura e distribuição espacial de uma população de Kielmeyera coriacea Mart. de cerrados de Brasília. Revista Brasileira de Botânica 12: $39-47$

Pereira, T.S. \& Mantovani, W. 2001. Maturação e dispersão de Miconia cinnamomifolia (DC.) Naud. na reserva biológica de Poço das Antas, município de Silva Jardim, RJ, Brasil. Acta Botanica Brasilica 15: 335-348.

Pizo, M.A. 1997. Seed dispersal and predation in two populations of $\mathrm{Ca}$ bralea canjerana (Meliaceae) in the Atlantic Forest of southeastern Brazil. Journal of Tropical Ecology 13: 559-578.

Ridley, H.N. 1930. The Dispersal of Plants Throughout the World London, Reeve.

Rizzini, C.T. 1987. Cactáceas. Os segredos da sobrevivência. Ciência Hoje 5: 30-40.

Rocha, C.F.D. \& Bergallo, H.G. 1994. Tropidurus torquatus (Collared lizard). Diet. Herpetological Review 25: 69.

Romão, R.L.; Hughes, F.M.; Vieira, A.M.C. \& Fontes, E.C. 2007. Autoecologia de Cabeça-de-frade (Melocactus ernestii Vaupel) em duas áreas de afloramentos na Bahia. Revista Brasileira de Biociências 5: 738-740.

Schupp, E.W. 1993. Quantity, quality and the effectiveness of seed dispersal by animals. Vegetatio 107/108: 15-29.

Taylor, N.P. \& Zappi, D.C. 2004. Cacti of eastern Brazil. Kew, Royal Botanic Gardens.

Taylor, N.P. 1991. The genus Melocactus (Cactaceae) in Central and South America. Bradleya 9: 1-80.

Taylor, N.P. 2000. Taxonomy and phytogeography of the Cactaceae of eastern Brazil. PhD, The Open University e Royal Gardens, Kew.

Teixeira, R.L. \& Giovanelli, M. 1999. Ecologia de Tropidurus torquatus (Sauria: Tropiduridae) da Restinga de Guriri, São Mateus, ES. Revista Brasileira de Biologia 59: 1-18 
Traveset, A. \& Verdú, M. 2002. A meta-analysis of the effect of gut treatment on seed germination. Pp. 339-350. In: D. J. Levey; W. R. Silva \& M. Galetti, (Eds.). Seed dispersal and frugivory: ecology, evolution and conservation. Wallingford, CAB International Publishing.

Valido, A. \& Nogales, M. 1994. Frugivory and seed dispersal by the lizard Gallotia galloti (Lacertidae) in a xeric habitat of the Canary Islands. Oikos 70: 403-411.
Van Der Pijl, L. 1982. Principles of dispersal in higher plants. 3. rd ed. Berlin, Springer-Verlag.

Vasconcellos-Neto, J.; Souza, A.L.T.; Guimarães, M.M. \& Faria, D.M. 2000. Effects of color, shape and location on detection of cactus fruits by a lizard. Journal of Herpetology 34: 306-309.

Vitt, L.J.; Zani, P.A. \& Caldwell, J.P. 1996. Behavioural ecology of Tropidurus hispidus on isolated rock outcrops in Amazônia. Journal of Tropical Ecology 12: 81-101. 